\title{
Vaccination for SARS-CoV-2 in Hematological Patients
}

\author{
Niccolò Riccardi ${ }^{a}$ Marco Falcone ${ }^{a}$ Dafna Yahav ${ }^{b, c}$ \\ aDepartment of Clinical and Experimental Medicine, Infectious Diseases Unit, Azienda Ospedaliera Universitaria \\ Pisana, University of Pisa, Pisa, Italy; 'bnfectious Diseases Unit, Rabin Medical Center, Beilinson Hospital, Petah-Tikva, \\ Israel; 'Sackler Faculty of Medicine, Tel Aviv University, Ramat Aviv, Israel
}

\section{Keywords}

Vaccinations $\cdot$ Severe acute respiratory syndrome coronavirus 2 - Hematological malignancies · Coronavirus disease 2019

\begin{abstract}
Patients with specific hematological malignancies (HM) are at increased risk for severe disease and death from infection with severe acute respiratory syndrome coronavirus 2 (SARSCoV-2). In healthy subjects, vaccination against SARS-CoV-2 has been demonstrated to be highly effective in disease prevention; however, immunocompromised patients were largely excluded from vaccine randomized controlled trials. In this review, we overview available non-randomized studies addressing effectiveness and safety of several coronavirus disease 2019 (COVID-19) vaccines in patients with HM. Overall, COVID-19 vaccines are safe in patients with $\mathrm{HM}$, with adverse events similar to those in the general population. Though serology testing is not recommended as a test to evaluate vaccine effectiveness, a correlation between higher antibody levels and protection against infection has been reported. Studies evaluating humoral response to COVID-19 vaccine in HM patients demonstrate low immunogenicity, mainly in patients with lymphoproliferative disorders, as well as with certain drugs, including mainly anti-CD20 antibodies, Bruton tyrosine kinase inhibitors, and also ruxolitinib
\end{abstract}

and venetoclax. Seropositivity rates of patients with nonHodgkin lymphoma and chronic lymphocytic leukemia following mRNA vaccination reach $40 \%-50 \%$. T-cell responses to vaccination are also impaired among these patients. Better humoral response rates are reported in multiple myeloma patients and hematopoietic stem-cell transplant, reaching $~ 75 \%-80 \%$, but not in patients following chimeric antigen receptor T-cell therapy. Patients with chronic myeloid leukemia and myeloproliferative diseases have high response rate to vaccination. Third mRNA vaccine dose is currently recommended to all $\mathrm{HM}$ patients. Alternative approaches for vaccination and prevention in patients unable to mount an immune response following full vaccination are provided in the review.

(c) 2022 S. Karger AG, Basel

\section{Introduction}

Patients with hematological malignancies (HM) have an increased risk for coronavirus disease 2019 (COVID-19) infection compared to the general population, with odds ratios of $\sim 12$ for leukemia patients and $\sim 8$ for non-Hodgkin lymphoma (NHL) patients [1]. Moreover, in these patients COVID-19 disease severity is increased, leading to hospitalizations and mortality. In addition, severe acute respiratory syndrome coronavirus 2 (SARS-
Karger@karger.com www.karger.com/aha
(C) 2022 S. Karger AG, Basel 
CoV-2) infection may also cause delays in chemotherapy, contributing to the poor outcome $[1,2]$. In healthy subjects, SARS-CoV-2 mRNA vaccines have demonstrated robust immune response and clinical efficacy in clinical trials, as well as considerable effectiveness in real life in the general population [3]. However, patients with HM were largely excluded from clinical vaccine trials or were poorly represented, and demonstrated relatively poor immunogenicity to these vaccines $[2,4]$. Immunogenicity varies depending on type of malignancy and therapy. Little is known about clinical effectiveness in HM patients. In this review, we aim to evaluate the available literature on immunogenicity and clinical effectiveness of different vaccines for various HMs, their safety, and the available strategies to improve immunogenicity in this population.

\section{Approved Vaccines for HM Individuals}

Vaccines based on live attenuated virus or replicating viral vectors (as vesicular stomatitis virus) are contraindicated for immunocompromised patients. None of these are currently approved for use $[5,6]$.

\section{Immune Response to Vaccines in HM}

Patients with HM have diminished immune response to vaccines in general [7] (Table 1). Reduced immunogenicity to SARS-CoV-2 vaccines have been suggested in $\mathrm{HM}$ as well, with several factors associated with this reduced response. In general, active immunosuppressive treatment is associated with lower anti-SARS-CoV-2 neutralizing antibody levels when tested compared to untreated patients [4]. Improved antibody response is demonstrated while vaccinated at least 6 months after the end of chemotherapy or hematopoietic stem-cell transplantation (HSCT), excluding anti-CD 20, for which antibody response improves significantly only at least 12 months after the end of therapy [4]. Other factors associated with blunted humoral response in HM patients in general include age $[4,8]$, male gender [9], CD19 + B cells levels $(<120 / \mu \mathrm{L})$ [9], and type of treatment, with Bruton tyrosine kinase inhibitors, ruxolitinib, venetoclax, and antiCD20 antibody therapies associated with most diminished response. In addition, waning antibody titers have been demonstrated in HM patients 4-6 months after vaccination, though most patients maintained detectable levels [10]. In regards to type of mRNA vaccine, several studies in immunocompromised patients demonstrated better antibody response with higher titers among patients receiving mRNA-1273 versus $\mathrm{BNT} 162 \mathrm{~b} 2$ vaccine [11-13].

Most studies evaluated humoral immune response; however, T-cell response has also a role in protecting against COVID-19 and has been demonstrated to confer improved survival in HM patients [14]. Few data are available regarding T-cell response to COVID-19 vaccine in HM patients. Malard et al. [9] demonstrated T-cell response in $53 \%(36 / 68)$ of mixed HM population in response to two BNT162b2 doses, and also found that this was achieved among 17 patients with no humoral response, suggesting some protective effect in these patients. Current treatment was associated with the absence of T-cell response in this study. Mairhofer et al. [15] reported either a CD4+ or a CD8+ T-cell response in $76 \%$ (34/35) patients with $\mathrm{HM}$, and a 58\% (26/45) antibody response in the same group. Notably, none of the patients treated with anti-CD20 achieved a "triple" response, of both antibodies, CD4+ and CD8+.

The response after one dose of mRNA vaccine is poor, leaving most patients practically unprotected. Hence, for HM patients, it is not recommended to prolong the interval between vaccine doses, rather consider adding further booster doses in order to increase immunogenicity [16, 17]. In this review, we will not consider vaccine efficacy after one dose, rather use the term "vaccination" as referring to at least two mRNA vaccine doses.

In general, it is not recommended to perform serological tests in order to determine whether the patient is protected [18]. Nevertheless, antibody levels are often used both in research and in clinical practice, and a correlation between higher antibody levels and neutralization of virus has been shown, though not in all studies $[19,20]$. We cannot provide any recommendation regarding use of serology to guide vaccination schedule in this review, and local guidelines on vaccination schedule should be followed.

\section{Safety of COVID-19 Vaccines in HM Patients}

As of September 2021, 392 million doses of BNT162b2 and over 54 million doses of mRNA-1723 were administered in European countries [21]. Safety of mRNA vaccines has been demonstrated to be similar in patients with HM compared to healthy individuals [16]. The most common adverse events among HM patients are sore arm and muscle aches [12]. Regarding the Oxford-AstraZeneca and Janssen/Johnson \& Johnson vaccines, safety has 


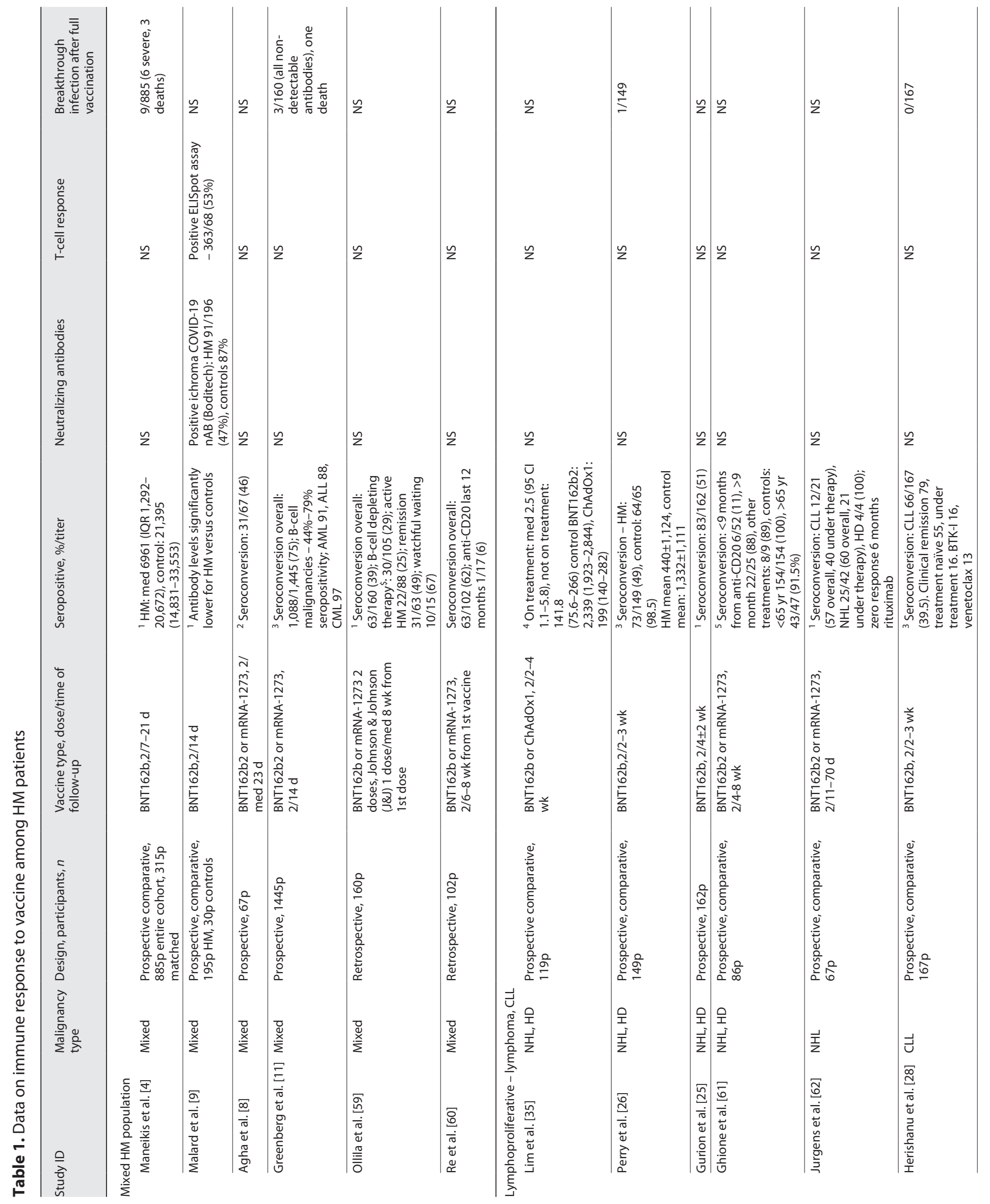




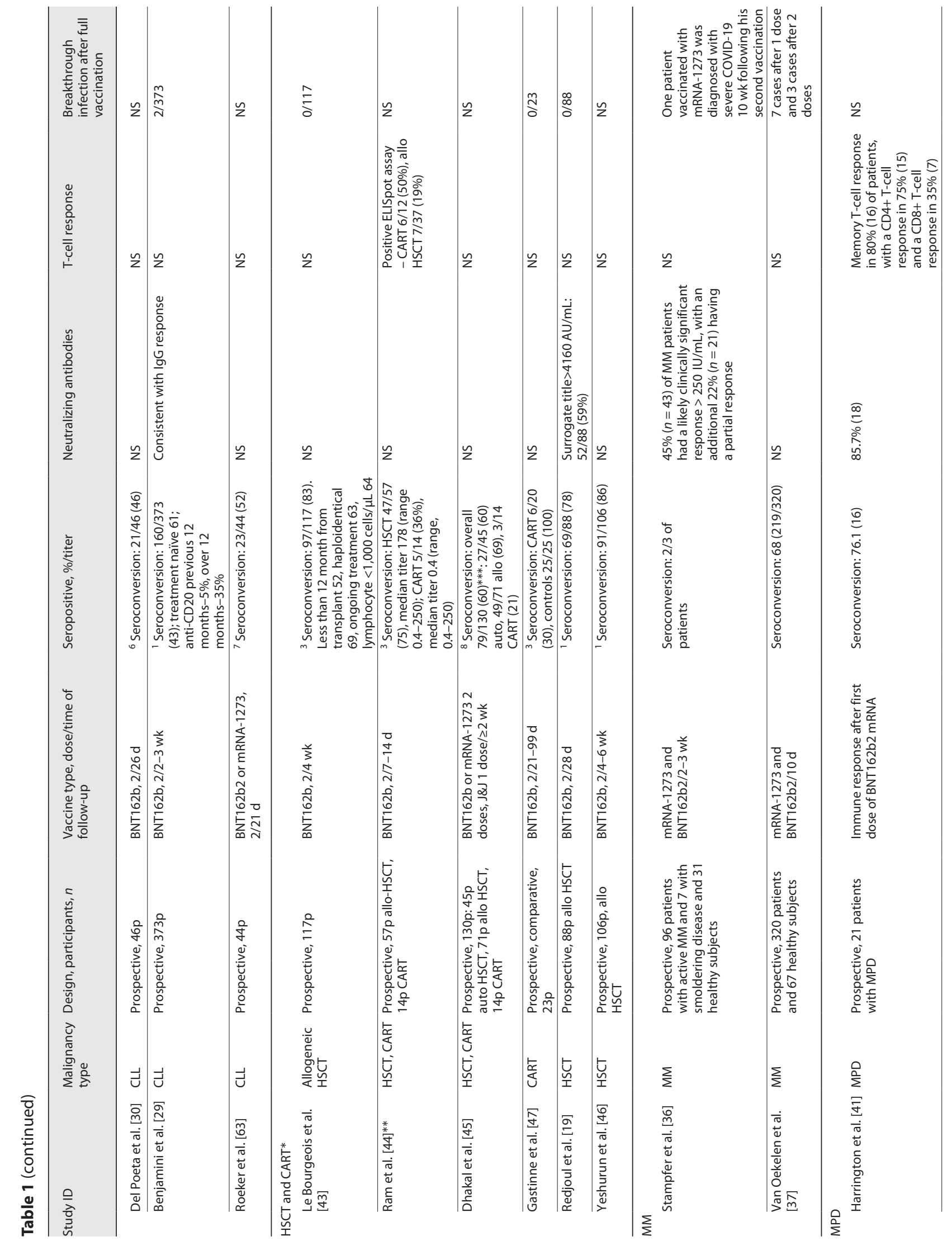




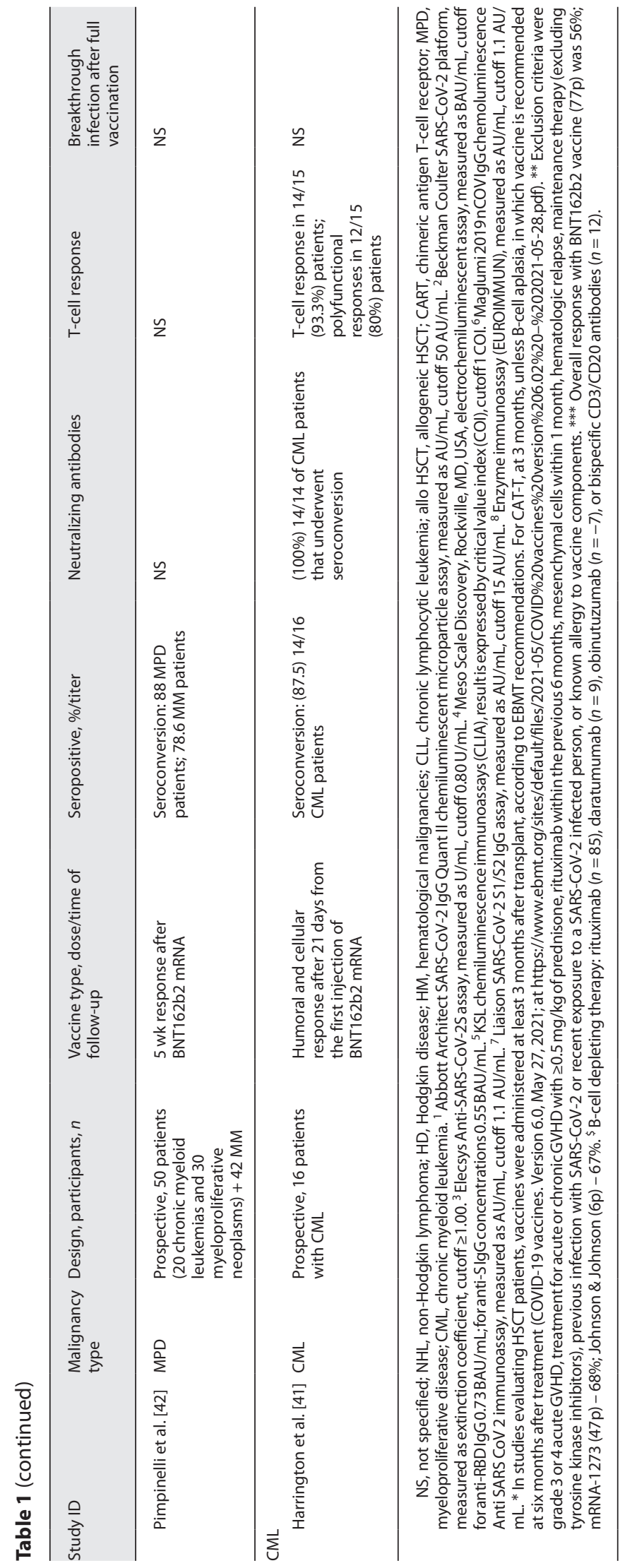

become a concern due to reports on vaccine-induced immune thrombocytopenia and thrombosis (also termed "thrombosis with thrombocytopenia syndrome") [22]. Due to this adverse event, some countries restrict these vaccines to the general population or based on age, though an overall total of 1,503 cases had been reported worldwide until July 31, 2021 [21].

\section{Immunogenicity of COVID-19 Vaccines in Specific HM}

As HM and their therapy are highly heterogeneous, so is the immune response to vaccines. In the following sections, we will review immune response and adverse events for several types of HM, and overview current recommendations for vaccination.

\section{Lymphoproliferative Disorders - Chronic Lymphocytic Leukemia, Lymphoma, and Anti-CD20 Therapy}

Patients with NHL and chronic lymphocytic leukemia (CLL) experience poor prognosis of COVID-19, with 30day mortality reaching $\sim 35 \%$ in unvaccinated patients $[23,24]$. Seropositivity rates following 2 doses of BNT162b2 among lymphoma patients are reported at $\sim 50 \%$ [25-27], and among CLL patients at 40\%-45\% [27-30]. Several predictors of seronegativity are described in the literature. These include the type of lymphoma aggressive NHL (63\% seronegativity) versus indolent NHL (54\%) versus Hodgkin disease, with low rates of seronegativity (10\%) in the latter [25]. Active therapy (considered until at least 6 months since discontinuation) is also associated with decreased antibody response and titers [26], depending on type and timing of therapy. Seropositivity rates for patients treated with anti-CD20 in the last 12 months are in most studies $0 \%-10 \%[4,26,28,29]$. These rates reach $25 \%-35 \%$ after over 12 months [26, 29], with one study even showing $68 \%$ response [31]. Median time from anti-CD20 to seropositivity was demonstrated in one study to be 36 months in indolent NHL and 20 months in aggressive NHL [26]. Gurion et al. [25] reported zero seroconversion among 28 patients treated with obinutuzumab, compared to $38 \%$ with rituximab.

Other predictors of negative response included older age ( $>80$ year [25], $>60$ [29]), low lymphocyte counts $\left(<0.9-1.2 \times 10^{3} / \mu \mathrm{L}\right)[25,26]$, hypogammaglobulinemia (below $630 \mathrm{~g} / \mathrm{L}$ ) [25, 28], male gender [28], IgM $<40 \mathrm{mg} /$ 
$\mathrm{dL}$, and $\operatorname{IgA}<80 \mathrm{mg} / \mathrm{dL}$ [29]. Benjamini et al. [29] suggested a model to predict immune response to vaccine among CLL patients, including recent anti-CD20 treatment, age $<70$ year, treatment naïve status, and normal IGG, IGA, IGM, and hemoglobin levels.

Few data are available regarding response to vaccines other than mRNA based, neutralizing antibody or T-cell responses, and seropositivity under therapies other than anti-CD20. Gavriatopoulou et al. [32] demonstrated neutralizing antibody response $\geq 50 \%$ (cutoff of clinically relevant inhibition) in $3 \%$ of 58 patients with NHL, Waldenstrom macroglobulinemia or CLL, compared to $24 \%$ response among controls, after one dose of either BNT162b2 or ChAdOx1 (AZD1222) vaccine. Terpos et al. [33] tested these values after BNT162b2 second dose, and found 44\% among 132 patients with CLL/lymphoma, compared to $95 \%$ in 214 controls. Only 7 patients on active treatment developed adequate levels of neutralizing antibodies. Liebers et al. [31] tested T-cell response to 2 mRNA vaccine doses among anti-CD20 treated lymphoma patients and found $58 \%$ response, compared to $71 \%$ in controls. Response rates were higher among patients who seroconverted and were not associated with time elapsed since the last anti-CD20 dose. Other studies also demonstrated lower, but existing $\mathrm{T}$-cell responses among seronegative lymphoma patients [34], suggesting a possible benefit of vaccination even in seronegative patients.

Lim et al. [35] demonstrated significantly reduced antibody titers for patients with various types of lymphoma compared to healthy controls, after 2 doses of either ChAdOx1 or BNT162b2. No significant difference was demonstrated between the two vaccines, though the small sample size was small (119 patients). Among patients on active treatment, $61 \%$ (20/33) did not seroconvert after 2 vaccine doses. In this study, patients with Hodgkin disease or aggressive B-cell NHL not on active treatment presented robust antibody responses, in contrast to patients with indolent B-cell NHL, who had reduced antibody levels [35].

Seropositivity under active BTK inhibitors therapy following 2 doses of mRNA vaccine is reported between $0 \%$ and $18 \%[4,28,29]$. Previous therapy is also associated with only $37 \%$ response. Treatment with ibrutinib was found to be significantly associated with no antibody response among CLL patients in a large series [29]. Venetoclax-including regimens were also associated with low response of $0 \%-24 \%$ seropositivity. Few data on ruxolitinib also demonstrated the absence of antibody response $[4,28]$.

Overall, patients with lymphoproliferative disease treated with either anti-CD-20, BTK-I, or BCL-2i are un- likely to produce adequate antibody response to mRNA vaccine and are probably not protected against SARSCoV-2 infection even after 2 vaccine doses. Healthcare workers who treat these patients should emphasize the importance of non-pharmacological protective measures for these patients (mainly social distancing, masks, and hand hygiene). Household members of these patients should be encouraged to vaccinate. Some have suggested weighing the risk/benefit ratio of anti-CD20 therapy in patients receiving anti-CD20 as maintenance therapy.

\section{Immune Response to SARS-CoV-2 Vaccines in Multiple Myeloma}

Antibody responses to the two mRNA vaccines against SARS-CoV-2, mRNA-1273, and BNT162b2 were assessed in an observational study by Stampfer et al. [36] in 103 patients with multiple myeloma (MM) compared to age-matched healthy subjects. Interestingly, MM patients with age $>65$ years; low absolute lymphocytes count; reduced levels of uninvolved IgG, IgA, IgM, or sFLC lambda; elevated creatinine levels ( $>1.3 \mathrm{mg} / \mathrm{dL}$ ); and current treatment with steroids had a lower antibody response. As expected, the more advanced the disease, the poorer the response to COVID-19 mRNA vaccination. Unfortunately, due to the heterogeneous MM treatments administered to the patients, no specific treatment could have been correlated with antibody response. More than half of MM patients in this cohort failed to fully respond to COVID-19 vaccination and the use of mRNA-1273 resulted in higher anti-spike antibody levels than BNT162b2 in this population. Van Oekelen et al. [37] reported higher response rates to 2 doses of $\mathrm{mRNA}$ vaccine in myeloma patients, with $68 \%(219 / 320)$ response, though 60 patients had COVID-19 prior to vaccination. Antibody levels achieved were significantly lower compared to healthy controls, and lower among patients treated with antiCD38 or anti-BCMA. Unsatisfactory immune response was also observed in $\mathrm{MM}$ patients vaccinated with ChAdOx1 (AZD1222) vaccine [38]. Aleman et al. [39] demonstrated variable cellular immune response to 2 doses of mRNA vaccine among MM patients that correlated with humoral response. Seropositive MM patients had similar CD4+ and CD8+ responses as healthy controls; however, low response rates $(35 \%$ for CD4+ and $28 \%$ for $\mathrm{CD} 8+$ ) were demonstrated among seronegative patients. The latters were more likely to be patients receiving anti-CD38 and anti-BCMA [39]. Variable immune responses among MM patients may be explained 
by the heterogeneity of patients - rates of hypogammaglobulinemia, disease severity, and type of therapy. Currently, the CDC recommends a booster third dose of mRNA vaccine in MM patients [5]. Of note, a fatal systemic capillary leak syndrome has been documented in a patient with $\mathrm{MM}$ after vaccination with the doublestranded DNA-Ad26.COV2.S vaccine [40].

\section{Immune Response to SARS-CoV-2 Vaccines in Myeloproliferative Disease}

A recent study by Harrington et al. [41] correlated similar immunological response after a single dose of $\mathrm{BNT} 162 \mathrm{~b} 2$ to the general population in patients with $\mathrm{my}-$ eloproliferative disease. In an Italian cohort of myeloproliferative disease patients, BNT162b2 full vaccination resulted in efficient protection after 5 weeks, except for patients with myelofibrosis, and with previous anti-CD38-based therapy [42].

\section{Immune Response to SARS-CoV-2 Vaccines in Chronic Myeloid Leukemia}

In contrast to patients with solid tumor or lymphoid $\mathrm{HM}$, a good immunogenicity to a single dose of the BNT162b2 vaccine has been noticed. Both T-cell and polyfunctional responses were produced, thus creating the bases for a sustained protection after the second dose and the possible boosting third dose [41].

\section{HSCT and Chimeric Antigen Receptor T-Cell Therapy}

\section{Immune Response to Vaccine}

Depending on time from transplant, patients following HSCT have considerable antibody responses, relative to other HM. Vaccination at least 3 months after transplantation resulted in $75 \%-83 \%$ seroconversion among patients after allogeneic HSCT [19, 43, 44]. Le Bourgeois et al. [43] reported that $62 \%$ of allogeneic HSCT recipients reached the highest IgG titer of the relevant assay. Factors associated with lower antibody response rates in studies included time from transplantation (6-12 months cutoffs); haploidentical donor; acute or chronic graft-versus-host disease (GVHD); current or within 3 months of vaccine of systemic immunosuppressive treatment; and total lymphocyte count $(<1,000$ cells/ $\mu \mathrm{L})[19,43,45,46]$.
Antibody response in patients after chimeric antigen receptor T-cell (CART) infusion is reported to be $21 \%$ $36 \%[44,45,47]$. Among a population of allogenic HSCT and CART patients, longer time from transplant, female sex, and higher CD19 + cells were associated with improved immune response to $\mathrm{BNT} 162 \mathrm{~b} 2$ vaccine in one study [44].

T-cell response to BNT162B2 was evaluated by Ram et al. [44], demonstrating positive response in 50\% CART patients evaluated (6/12) and 19\% (7/37) after allogeneic HSCT. Either serology or T-cell response (evaluated by ELISpot test) was positive in 57\% after CART infusion and $75 \%$ after allogeneic HSCT. The latter finding of high rates of T-cell response in HSCT patients is further supported by a recent study demonstrating over $80 \%$ of $\mathrm{T}$ cell response after 2 mRNA vaccine doses, with high rates of polyfunctional T-cell response, assumed to provide more effective antiviral effect [48]. Though adequate antibody response is achieved in most HSCT patients, T-cell dysfunction expected in these patients may compromise overall immune response to vaccine, and further studies should explore T-cell immunity in these patients.

\section{Safety in HSCT}

Ram et al. [44] reported no grade 3 or 4 non-hematologic adverse events among 57 allogeneic HSCT recipients receiving 2 BNT162b2 doses; however, 4 patients $(5 \%)$ developed grade 3 or 4 thrombocytopenia $(n=3)$ or neutropenia $(n=1)$ in this cohort, one impending secondary rejection event was considered to be possibly related to vaccine; and 3 cases of GVHD exacerbation were reported in this study after each vaccine dose $(\sim)$, all easily controlled. Yeshurun et al. [46] also reported worsening of GVHD symptoms following vaccination in $7 \%$ of patients. Ali et al. [49] reported new chronic GVHD in $\sim 10 \%$ and worsening chronic GVHD in $3.5 \%$ of 113 allogeneic HSCT recipients who received at least one mRNA vaccine dose. Grade 3-4 thrombocytopenia ( $n=$ $4)$ or neutropenia $(n=4)$ were also reported.

\section{Specific Society Recommendations}

According to EBMT recommendations, any non-liveattenuated vaccine can be used; however, vaccines that showed $a \geq 90 \%$ response in phase 3 trials are preferable, due to the expected relatively low response in HSCT/ CART. Recommended timing of vaccination in case the transmission rate in the community is high, is 3 months after HSCT, otherwise -6 months. Patients receiving their COVID-19 vaccine prior to transplantation should be revaccinated. EBMT recommendation is to prioritize 
the COVID-19 vaccine over the regular vaccinations program. Current recommendation is to administer this vaccine alone and to avoid other vaccines within 14 days of administration. EBMT suggests possible criteria to postpone the vaccine, including GVHD grade III-IV, antiCD20 past 6 months, CART with B-cell aplasia within 6 months of infusion, recent ATG, or alemtuzumab [50].

\section{Vaccine-Induced Immune Thrombocytopenia and Thrombosis}

Along with the unprecedented worldwide administration of anti-SARS-CoV-2 vaccines, new side effects, previously not reported in clinical trials, have emerged. The ChAdOx1 nCoV-19 adenoviral vector vaccine has been associated with vaccine-induced immune thrombocytopenia and thrombosis, a clinical syndrome characterized by thrombosis at unusual sites, thrombocytopenia, elevated D-dimer levels, high-titer antibodies to platelet factor 4 , and reduced fibrinogen levels in young patients (mainly age $<60$ years) and with high mortality $[51,52]$.

\section{Timing of Vaccination}

The recommendation of the American Society of Hematology regarding vaccinating persons who are less likely to respond to the vaccine is to weigh the decision on a case-by-case basis. It is recommended to consider both the incidence of SARS-CoV-2 infection in the community, type of vaccine available, and patient characteristics (underlying disease and level of immunosuppression). Recommended timing of vaccination is either 2-4 weeks prior to cancer therapy, or 6 months after the end of cancer therapy. However, if community transmission is high, it is reasonable to administer the vaccine earlier, even if it is expected to provide lower protection rates [53]. The European society of hematology recommends an interval of 3-6 months in analogy to other vaccinations [54].

\section{Third mRNA Dose}

In August 2021, the US Food and Drug Administration (FDA) authorized the third mRNA vaccine dose of both BNT162b2 and mRNA-1273 for immunocompromised patients. These include individuals on active chemotherapy, HSCT, and immunosuppressive therapy (e.g., anti CD20, other biologic agents, antimetabolites, alkylating agents, prednisone $\geq 20 \mathrm{mg}$ daily). Several studies in kidney transplant recipients demonstrated improved humoral and cellular immunogenicity one month following a third mRNA vaccine dose, supporting FDA's decision [55]. In light of these findings, the Advisory Committee on Immunization Practices also recommends an additional dose of either mRNA vaccine (BNT162b2 for persons aged $\geq 12$ years or mRNA 1273 for persons aged $\geq 18$ years) at least 28 days after an initial 2 -dose regimen for patients who are moderately to severely immunocompromised. Both FDA and CDC do not recommend testing serologic or cellular response for the decision on an additional vaccine dose, due to lack of data to support this practice. Additional third vaccine dose is recommended by the CDC for immunocompromised individuals regardless of prior SARS-CoV-2 infection. It is also recommended to administer the third vaccine dose at least 90 days after passive antibody therapy (monoclonal antibodies or convalescent plasma) $[5,56]$. Initial reports in HM show good response rates, with one study reporting seroconversion of over half of HM patients seronegative prior to the third dose. Anti-CD20 and BTK-I therapy were associated with lower rates of seroconversion and lower titer [10]. Fourth mRNA vaccine dose has been already administered in solid organ transplant recipients, showing good response for those with previous antibody response, and limited response among those who were seronegative after the third dose [57].

\section{Conclusions}

Patients with HM present diminished immune response to current vaccination strategies for COVID-19, as detailed above. Strategies to improve immunogenicity should be considered, which inclde the following:

1. Timing of vaccine - responses to vaccines were improved when administered later after HSCT or antiCD20. Delays in vaccination schedule may be considered at times of low community transmission of the virus, however, not during local outbreaks.

2. Booster doses - third mRNA vaccine dose is currently approved by the FDA for immunocompromised patients.

3. Heterologous vaccination - Maneikis et al. [4] demonstrated poor response to the second BNT162b2 dose in patients responding poorly to the first dose of the same vaccine. In solid organ transplant recipients, a third homologous or heterologous vaccination resulted in a modest response, with $25 \%$ of patients who were seronegative after two doses, mounting an immune response after a third dose [55].

For patients with no antibody response to vaccines, pre-exposure monoclonal antibodies, administered routinely as prophylaxis at times of outbreaks, should be considered [58]. 


\section{Conflict of Interest Statement}

The authors have no conflicts of interest to declare.

\section{Funding Sources}

None.

\section{References}

1 Wang Q, Berger NA, Xu R. Analyses of risk, racial disparity, and outcomes among US patients with cancer and COVID-19 infection. JAMA Oncol. 2021 Feb 1;7(2):220-7.

2 Kamboj M. Blunted humoral response after mRNA vaccine in patients with haematological malignancies. Lancet Haematol. 2021 Aug;8(8):e540-2.

3 Dagan N, Barda N, Kepten E, Miron O, Perchik S, Katz MA, et al. BNT162b2 mRNA covid-19 vaccine in a nationwide mass vaccination setting. N Engl J Med. 2021 Apr 15; 384(15):1412-23.

4 Maneikis K, Šablauskas K, Ringelevičiūtė U, Vaitekènaitė V, Čekauskienè R, Kryžauskaitė $\mathrm{L}$, et al. Immunogenicity of the BNT162b2 COVID-19 mRNA vaccine and early clinical outcomes in patients with haematological malignancies in Lithuania: a national prospective cohort study. Lancet Haematol. 2021 Aug;8(8):e583-92.

5 Centers for Disease Control and Prevention (CDC). Interim clinical considerations for use of mrna covid-19 vaccines currently authorized in the united states; 2021. Available from: https://www.cdc.gov/vaccines/covid-19/info-by-product/clinical-considerations.html.

6 Mikulska M, Cesaro S, de Lavallade H, Di Blasi R, Einarsdottir S, Gallo G, et al. Vaccination of patients with haematological malignancies who did not have transplantations: guidelines from the 2017 European conference on infections in leukaemia (ECIL 7). Lancet Infect Dis. 2019 Jun;19(6):e188-99.

7 La Torre G, Mannocci A, Colamesta V, D'Egidio V, Sestili C, Spadea A. Influenza and pneumococcal vaccination in hematological malignancies: a systematic review of efficacy, effectiveness, and safety. Mediterr J Hematol Infect Dis. 2016;8(1):e2016044.

8 Agha ME, Blake M, Chilleo C, Wells A, Haidar G. Suboptimal response to coronavirus disease 2019 messenger RNA vaccines in patients with hematologic malignancies: a need for vigilance in the postmasking era. Open Forum Infect Dis. 2021 Jul;8(7):ofab353.

9 Malard F, Gaugler B, Gozlan J, Bouquet L, Fofana D, Siblany L, et al. Weak immunogenicity of SARS-CoV-2 vaccine in patients with hematologic malignancies. Blood Cancer J. 2021 Aug 10;11(8):142.

10 Shapiro LC, Thakkar A, Campbell ST, Forest SK, Pradhan K, Gonzalez-Lugo JD, et al. Ef-

\section{Author Contributions}

All authors contributed to the planning of this review and writing of the manuscript. All authors reviewed and approved the final version. ficacy of booster doses in augmenting waning immune responses to COVID-19 vaccine in patients with cancer. Cancer Cell. 2021 Nov 16.

11 Greenberger LM, Saltzman LA, Senefeld JW, Johnson PW, DeGennaro LJ, Nichols GL. Antibody response to SARS-CoV-2 vaccines in patients with hematologic malignancies. Cancer Cell. 2021 Aug 9;39(8):1031-3.

12 Thakkar A, Gonzalez-Lugo JD, Goradia N, Gali R, Shapiro LC, Pradhan K, et al. Seroconversion rates following COVID-19 vaccination among patients with cancer. Cancer Cell. 2021 Aug 9;39(8):1081-90.

13 Boyarsky BJ, Werbel WA, Avery RK, Tobian AAR, Massie AB, Segev DL, et al. Antibody Response to 2-dose SARS-CoV-2 mRNA vaccine series in solid organ transplant recipients. JAMA. 2021 May 5.

14 Bange EM, Han NA, Wileyto P, Kim JY, Gouma S, Robinson J, et al. CD8+ T cells contribute to survival in patients with COVID-19 and hematologic cancer. Nat Med. 2021 Jul; 27(7):1280-9.

15 Mairhofer M, Kausche L, Kaltenbrunner S, Ghanem R, Stegemann M, Klein K, et al. Humoral and cellular immune responses in SARS-CoV-2 mRNA-vaccinated patients with cancer. Cancer Cell. 2021 Sep 13;39(9): $1171-2$.

16 Monin L, Laing AG, Muñoz-Ruiz M, McKenzie DR, Del Molino Del Barrio I, Alaguthurai $T$, et al. Safety and immunogenicity of one versus two doses of the COVID-19 vaccine BNT162b2 for patients with cancer: interim analysis of a prospective observational study. Lancet Oncol. 2021 Jun;22(6):765-78.

17 Re D, Seitz-Polski B, Carles M, Brglez V, Graça D, Benzaken S, et al. Humoral and cellular responses after a third dose of BNT162b2 vaccine in patients treated for lymphoid malignancies; 2021. Available from: https://www. medrxiv.org/content/10.1101/2021.07.18.21 260669v1.

18 Young J-AH. Beginning to understand clinical events and immune responses of hematopoietic cell transplant recipients receiving SARS-CoV-2 vaccination. Transplant Cell Therapy. 2021 Sep;27(9):700-1.

19 Redjoul R, Le Bouter A, Beckerich F, Fourati S, Maury S. Antibody response after second BNT162b2 dose in allogeneic HSCT recipients. Lancet. 2021 Jul 24;398(10297): 298-9.
20 Yamamoto S, Maeda K, Matsuda K, Tanaka A, Horii K, Okudera K, et al. COVID-19 breakthrough infection and post-vaccination neutralizing antibody among healthcare workers in a referral hospital in Tokyo: a casecontrol matching study. Clin Infect Dis. 2021 Dec 24:ciab1048.

21 Buckingham L. Safety of COVID-19 vaccines: European Medicines Agency; 2021. Available from: https://www.ema.europa.eu/en/human-regulatory/overview/public-healththreats/coronavirus-disease-covid-19/treatments-vaccines/vaccines-covid-19/safety-covid-19-vaccines.

22 Platton S, Bartlett A, MacCallum P, Makris M, McDonald V, Singh D, et al. Evaluation of laboratory assays for anti-platelet factor 4 antibodies after ChAdOx1 nCOV-19 vaccination. J Thromb Haemost JTH. 2021 Aug;19(8): 2007-13.

23 Regalado-Artamendi I, Jiménez-Ubieto A Hernández-Rivas JÁ, Navarro B, Núñez L, Alaez C, et al. Risk Factors and Mortality of COVID-19 in patients with lymphoma: a multicenter study. HemaSphere. 2021 Mar; 5(3): 5338

24 Mato AR, Roeker LE, Lamanna N, Allan JN, Leslie L, Pagel JM, et al. Outcomes of COVID-19 in patients with CLL: a multicenter international experience. Blood. 2020 Sep 3; 136(10):1134-43.

25 Gurion R, Rozovski U, Itchaki G, Gafter-Gvili A, Leibovitch C, Raanani P, et al. Humoral serologic response to the BNT162b2 vaccine is abrogated in lymphoma patients within the first 12 months following treatment with antiCD2O antibodies. Haematologica. $2021 \mathrm{Jul}$ 29.

26 Perry C, Luttwak E, Balaban R, Shefer G, Morales MM, Aharon A, et al. Efficacy of the BNT162b2 mRNA COVID-19 vaccine in patients with B-cell non-Hodgkin lymphoma. Blood Adv. 2021 Aug 24;5(16): 3053-61.

27 Ribas A, Dhodapkar MV, Campbell KM, Davies FE, Gore SD, Levy R, et al. How to provide the needed protection from COVID-19 to patients with hematologic malignancies. Blood Cancer Discov. 2021 Nov;2(6):562-7.

28 Herishanu Y, Avivi I, Aharon A, Shefer G, Levi S, Bronstein Y, et al. Efficacy of the BNT162b2 mRNA COVID-19 vaccine in patients with chronic lymphocytic leukemia. Blood. 2021 Jun 10;137(23):3165-73. 
29 Benjamini O, Rokach L, Itchaki G, Braester A, Shvidel L, Goldschmidt N, et al. Safety and efficacy of BNT162b mRNA Covid19 Vaccine in patients with chronic lymphocytic leukemia. Haematologica. 2021 Jul 29.

30 Del Poeta G, Bomben R, Polesel J, Rossi FM, Pozzo F, Zaina E, et al. COVID-19 vaccination: evaluation of risk for protection failure in chronic lymphocytic leukemia patients. Hematol Oncol. 2021 Aug 30.

31 Liebers N, Speer C, Benning L, Bruch P-M, Krämer I, Meissner J, et al. Humoral and cellular responses after COVID-19 vaccination in anti-CD20 treated lymphoma patients. Blood. 2021 Oct 20.

32 Gavriatopoulou M, Terpos E, Kastritis E, Briasoulis A, Gumeni S, Ntanasis-Stathopoulos I, et al. Low neutralizing antibody responses in WM, CLL and NHL patients after the first dose of the BNT162b2 and AZD1222 vaccine. Clin Exp Med. 2021 Jul 20.

33 Terpos E, Gavriatopoulou M, Fotiou D, Giatra C, Asimakopoulos I, Dimou M, et al. Poor Neutralizing antibody responses in $132 \mathrm{pa}-$ tients with CLL, NHL and HL after vaccination against SARS-CoV-2: a prospective study. Cancers. 2021 Sep 6;13(17):4480.

34 Marasco V, Carniti C, Guidetti A, Farina L, Magni M, Miceli R, et al. T-cell immune response after mRNA SARS-CoV-2 vaccines is frequently detected also in the absence of seroconversion in patients with lymphoid malignancies. Br J Haematol. 2021 Oct 14.

35 Lim SH, Campbell N, Johnson M, JosephPietras D, Collins GP, O'Callaghan A, et al. Antibody responses after SARS-CoV-2 vaccination in patients with lymphoma. Lancet Haematol. 2021 Aug;8(8):e542-4.

36 Stampfer SD, Goldwater M-S, Jew S, Bujarski S, Regidor B, Daniely D, et al. Response to mRNA vaccination for COVID-19 among patients with multiple myeloma. Leukemia. 2021 Jul 29.

37 Van Oekelen O, Gleason CR, Agte S, Srivastava K, Beach KF, Aleman A, et al. Highly variable SARS-CoV-2 spike antibody responses to two doses of COVID-19 RNA vaccination in patients with multiple myeloma. Cancer Cell. 2021 Aug 9;39(8):1028-30.

38 Bird S, Panopoulou A, Shea RL, Tsui M, Saso $\mathrm{R}$, Sud A, et al. Response to first vaccination against SARS-CoV-2 in patients with multiple myeloma. Lancet Haematol. 2021 Jun; 8(6):e389-92.

39 Aleman A, Upadhyaya B, Tuballes K, Kappes $\mathrm{K}$, Gleason CR, Beach K, et al. Variable cellular responses to SARS-CoV-2 in fully vaccinated patients with multiple myeloma. Cancer Cell. 2021 Nov 8;39(11):1442-4.

40 Choi GJ, Baek SH, Kim J, Kim JH, Kwon GY, Kim DK, et al. Fatal systemic capillary leak syndrome after SARS-CoV-2 vaccination in patient with multiple myeloma. Emerg Infect Dis. 2021 Nov; 27(11):2973-5.

41 Harrington P, de Lavallade H, Doores KJ, O’Reilly A, Seow J, Graham C, et al. Single dose of BNT162b2 mRNA vaccine against
SARS-CoV-2 induces high frequency of neutralising antibody and polyfunctional T-cell responses in patients with myeloproliferative neoplasms. Leukemia. 2021 May 22.

42 Pimpinelli F, Marchesi F, Piaggio G, Giannarelli D, Papa E, Falcucci P, et al. Fifthweek immunogenicity and safety of antiSARS-CoV-2 BNT162b2 vaccine in patients with multiple myeloma and myeloproliferative malignancies on active treatment: preliminary data from a single institution. $\mathrm{J} \mathrm{He}$ matol Oncol. 2021 May 17;14(1):81.

43 Le Bourgeois A, Coste-Burel M, Guillaume T, Peterlin P, Garnier A, Béné MC, et al. Safety and antibody response After 1 and 2 doses of BNT162b2 mRNA vaccine in recipients of allogeneic hematopoietic stem cell transplant. JAMA Netw Open. 2021 Sep 1;4(9):e2126344.

44 Ram R, Hagin D, Kikozashvilli N, Freund T, Amit O, Bar-On Y, et al. Safety and immunogenicity of the BNT162b2 mRNA COVID-19 vaccine in patients after allogeneic HCT or CD19-based CART therapy: a single-center prospective cohort study. Transplant Cell Ther. 2021 Sep;27(9):788-94.

45 Dhakal B, Abedin S, Fenske T, Chhabra S, Ledeboer N, Hari P, et al. Response to SARS$\mathrm{CoV}-2$ vaccination in patients after hematopoietic cell transplantation and CAR T-cell therapy. Blood. 2021 Oct 7;138(14):1278-81.

46 Yeshurun M, Pasvolsky O, Shargian L, Yahav D, Ben-Zvi H, Rubinstein M, et al. Humoral serologic response to the BNT162b2 vaccine after allogeneic haematopoietic cell transplantation. Clin Microbiol Infect. 2021 Oct 26.

47 Gastinne T, Le Bourgeois A, Coste-Burel M, Guillaume T, Peterlin P, Garnier A, et al. Safety and antibody response after one and/or two doses of BNT162b2 Anti-SARS-CoV-2 mRNA vaccine in patients treated by CAR T cells therapy. Br J Haematol. 2021 Sep 2.

48 Harrington P, Doores KJ, Saha C, Saunders J, Child F, Dillon R, et al. Repeated vaccination against SARS-CoV-2 elicits robust polyfunctional $\mathrm{T}$ cell response in allogeneic stem cell transplantation recipients. Cancer Cell. 2021 Nov 8;39(11):1448-9.

49 Ali H, Ngo D, Aribi A, Arslan S, Dadwal S, Marcucci G, et al. Safety and tolerability of SARS-CoV2 emergency-use authorized vaccines for allogeneic hematopoietic stem cell transplant recipients. Transplant Cell Ther. 2021 Nov;27(11):938.e1-938.e6.

50 European Society for Blood and Marrow Transplantation. COVID-19 vaccines. Version $6.0 ; 2021$. Available from: https://www. ebmt.org/sites/default/files/2021-05/ COVID\%20vaccines\%20version\%206.02\%20 -\%202021-05-28.pdf.

51 Schultz NH, Sørvoll IH, Michelsen AE Munthe LA, Lund-Johansen F, Ahlen MT, et al. Thrombosis and thrombocytopenia after ChAdOx1 nCoV-19 vaccination. N Engl J Med. 2021 Jun 3;384(22):2124-30.

52 Greinacher A, Thiele T, Warkentin TE, Weisser K, Kyrle PA, Eichinger S. Thrombot- ic Thrombocytopenia after ChAdOx1 nCov19 vaccination. N Engl J Med. 2021 Jun 3; 384(22):2092-101.

53 ASH-ASTCT COVID-19 and Vaccines: Frequently Asked Questions - Hematology.org [Internet]. [cited 2021 Nov 2]. Available from: https: //www.hematology.org: 443/covid-19/ ash-astct-covid-19-and-vaccines.

54 European Hematology Association. Expert opinions for COVID-19 vaccination in patients with hematologic cancer: The European Hematology Association; 2021. Available from: https://ehaweb.org/covid-19/eha-statement-on-covid-19-vaccines/recommendations-for-covid19-vaccination-in-patients-with-hematologic-cancer/.

55 Werbel WA, Boyarsky BJ, Ou MT, Massie AB, Tobian AAR, Garonzik-Wang JM, et al. Safety and immunogenicity of a third dose of SARS-CoV-2 vaccine in solid organ transplant recipients: a case series. Ann Intern Med. 2021 Sep;174(9):1330-2.

56 Centers for Disease Control. Immunocompromised patients \& COVID-19 vaccine: Centers for Disease Control and Prevention; 2021. Available from: https://www.cdc.gov/ vaccines/covid-19/clinical-considerations/ immunocompromised.html.

57 Alejo JL, Mitchell J, Chiang TP-Y, Abedon AT, Boyarsky BJ, Avery RK, et al. antibody response to a fourth dose of a SARS-CoV-2 vaccine in solid organ transplant recipients: a case series. Transplantation. 2021 Dec 1; 105(12):e280-1.

58 Astrazeneca. AZD7442 PROVENT Phase III prophylaxis trial met primary endpoint in preventing COVID-19; 2021. Available from: https: //www.astrazeneca.com/media-centre/ press-releases/2021/azd7442-prophylaxistrial-met-primary-endpoint.html.

59 Ollila TA, Lu S, Masel R, Zayac A, Paiva K, Rogers RD, et al. Antibody response to COVID-19 vaccination in adults with hematologic malignant disease. JAMA Oncol. 2021 Nov 1;7(11):1714-6.

60 Re D, Barrière J, Chamorey E, Delforge M, Gastaud L, Petit E, et al. Low rate of seroconversion after mRNA anti-SARS-CoV-2 vaccination in patients with hematological malignancies. Leuk Lymphoma. 2021 Jul 26: $1-3$.

61 Ghione P, Gu JJ, Attwood K, Torka P, Goel S, Sundaram S, et al. Impaired humoral responses to COVID-19 vaccination in patients with lymphoma receiving B-cell-directed therapies. Blood. 2021 Sep 2;138(9):811-4.

62 Jurgens EM, Ketas TJ, Zhao Z, Joseph Satlin M, Small CB, Sukhu A, et al. Serologic response to mRNA COVID-19 vaccination in lymphoma patients. Am J Hematol. $2021 \mathrm{Nov}$ 1;96(11):E410-3.

63 Roeker LE, Knorr DA, Thompson MC, Nivar M, Lebowitz S, Peters N, et al. COVID-19 vaccine efficacy in patients with chronic lymphocytic leukemia. Leukemia. 2021 Sep;35(9): 2703-5. 